\title{
INTEGRATING QUALITATIVE REASONING AND TEXT PLANNING TO GENERATE CAUSAL EXPLANATIONS
}

\author{
Farid CERBAH \\ DEA/IA \\ Dassault Aviation \\ 78, quai Marcel Dassault 92214 St-Cloud FRANCE \\ Tel: (33-1) 47115300 ; Fax: (33-1) 47115283 \\ Université de Caen
}

\section{$I-I N T R O D U C T I O N$}

Several works [McKeown 86, Suthers 88] have emphasized the common aspects of Explanation Production in expert systems and Text Gencration. The work described in this paper deals with text generation applied to a particular type of explanations: causal explanations of physical systems. They have already motivated influential developments in the field of qualitative reasoning about physical systems. A central goal of the theories developed in [De Kleer 84] and [Forbus 84] was to elaborate conceptual frameworks for providing causal accounts of physical systems, sensitive to our commonsense understanding of the physical world. Those qualitative causal models constitute an adequate starting point as we are interested in how people present such causal explanations in verbal form.

We will describe our approach for text generation, based on the study of texts collected in encyclopedia and textbooks, and currently developed in a sysiem intended to be associated to the qualitative simulation system SQUALE [Jézéquel \& Zimmer 92]. Our conceptual model, which constitutes the input to the text generation process, is based on Qualitative Process Theory [Forbus 84].

According to the "traditional" division of tasks in text generation, the transition from conceptual representation of causal behaviour to causal explanation in natural language is viewed as a three-stage process: content specification, text organization and surface generation.

The content specification task aims at posting communicative goals described by means of communicative acts on conceptual entities. In particular, the causal explanation to be produced is often restricted to some particular events of the causal behaviour. We will show how relevant information and appropriate communicative acts are identificd.

Text organization is the most claboratc part of our model and is also divided into three tasks.
The first is concerned with the construction of a textual structure from a set of communicative acts cstablished during content specification. This structure, which takes an intermediary place between communicative acts and surface realizations, specifies essentially prescriptions on grouping and ordering of textual units. This process is achieved through the application of discourse strategies which control local transitions from communicative acts to possible organizational prescriptions. We describe three strategies used for structuring causal explanations: a causal chain strategy (for organizing simple causal chains), a parallel strategy (to impose a parallel structure on the text), and $a$ concessive strategy (for performing concessive acts). The second task segments the textual structure into sentential contents. Several factors are involved, mainly communicative form of textual relations and number of textual units expressed in a sentence. The stylistic adjustment task deals with stylistic considerations related to lexico-syntactic choices. We want to ensure an inter-sentential compatibility, from a stylistic point of view, between linguistic realizattions.

Concerning surfacc gencration, two types of systems have been considered: a sentence generator [Fournier 91 ] based on the Meaning-Text Theory (Mel'cuk 88a) and a syntactic component based on cquivalence classes between sentence structures.

Our approach suggests a clear separation of knowledge sources involved in each of these stages. We will start with a presentation of the conceptual model, where we propose a charactcrization of causal interactions between physical events (essentially, physical parameter changes). The next sections deal with the different stages of the text generation process. 


\section{II-THE CONCEPTUAL FRAMEWORK}

Qualitative Process Theory helps to capture commonsense knowledge implicit in mathematical models of traditional physics. Besides, it provides an ontological framework for expressing physical causality, by expliciting the physical mechanisms. In order to describe physical situations, two kinds of structures are used : individuals views, for representing physical objects and behavioural abstractions, and physical processes such as fluid and heat flows. Gradual parameters are associated to identified entities (individual views and processes) and causal dependencies are expressed by means of two types of qualitative relations: influences, for direct effects of processes, and qualitaive proportionalities, which propagate direct effects of processes on the other parameters. It follows that the initial cause of a parameter change is always a process.

Qualitative relations can aim at opposite effects. In the physical situation of figure 1 , the mercury expansion tends to increase the mercury level while the tube expansion tends to lower it. The first influence is predominant, since an increase of the mercury level is observed in that situation, and thus the second is inhibited ${ }^{l}$. In order to represent different types of causal interactions, we have introduced the causal primitives cause, contrary-cause and oppositeeffects which are defined according to influences between events:

- cause: causal relation between two events linked with a predominant influence (for example, mercury expansion is the cause of mencury level rise)

- contrary-cause: contrastive relation between two events linked with an inhibited influence. One of the events is the "contrary cause" of the other (tube expansion is a contrary cause of mercury level rise).

- opposite-effects: contrastive relation between two events which aim at opposite effects (mercury expansion and tube expansion).

The last two primitives are called causal opposition relalions. In that framework, physical system behaviours arc represented with influence graphs, where physical events are linked with causal primitives. An influence graph $\mathrm{cn}-$ closes all events and causal interactions identified in the given physical situation.

\section{III - CONTENT SPECIFICATION}

The content specification is primarily concerned with selection of relevant physical events and causal interactions from the influence graph. But the problem should not be narrowed to factual knowledge selcction, for the conceptu-

1. It does not mean that the mercury expansion has no effect on the mercury level but that no effoet is perceived at that level of abstraction. al content of the text cannot be considered independently of the speaker's communicative intentions. Communicative roles assigned to conceptual entities have crucial implications at each stage of the text generation process. Hence, they should be made explicit at the content specification level as well as their related conceptual units. In our model, the content specification task results in a set of communicative acts, which represents the illocutionary structure of the messagc. These acts are performed so as to produce some effects on the hearer, as modifying his belief system or affecting his emotional states. They may be characterized according to these intended effects. We focus here on three types of acts: inform, explain and concede, and especially in the context of causal explanation production. Roughly speaking, an act of informing aims at making the hearer believe some proposition while an act of explaining, viewed as a discourse act, has the effect of supporting a previously introduced proposition in order to favour its acceptance. (1) and (2) are respective realizations of acts of informing and explaining:

(1) La dilatation du mercure provoque la hausse du niveau de mercure.

(The mercury expansion causes the rise of the mercury level.)

(2) Le niveau de mercure monte, parce que le mercure se dilate.

(The mercury level rises, because of the mercury expansion.)

(1) is a possible achievement of the act:

inform (cause(Ds/volume(mercury) $]=1$, Ds $($ level(mercury $)]=1))^{2}$

and (2), of both acts:

inform(Ds[level(mercury)] $=1)$ )

explain(Ds[level(mercury)] $=1$, Ds $($ volume $($ mercury $)]=1)$

In terms of speech acts, (1) is a single assertion, which informs the hcarer of the causal connection between the two events. In contrast, (2) contains two assertions, the second assuming an explicative function. Note that in both cases the same conceptual content is involved. Some differences between sentences (1) and (2) as the order of information units are due to distinet communicative role assignments. We will show in the next section how these intended "rhetorical" effects influcnce choices at the text organization level.

But now let us turn to the content specification procedures. The determination of communicative acts highly depends on the problem solving activity (here, qualitative simula-

2. In the formalism of Qualitative Process Theory, the Ds function provides the derivative sign of the parameter given as argument. $\mathrm{Ds}[\mathrm{X}]=-1$ means that $\mathrm{X}$ decreases, $\mathrm{Ds}[\mathrm{X}]=0$ that $X$ is steady and $D s[X]=1$ that $X$ increases. 


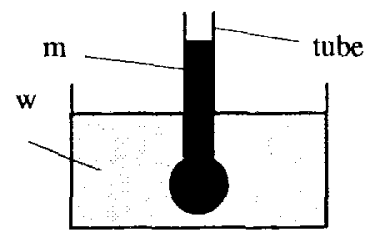

contained-liquid( $m$, sube, mercury)

liquid(w, water)

heat flow(hl, w, tube)

heat-flow $(h 2$, tube, $m)$

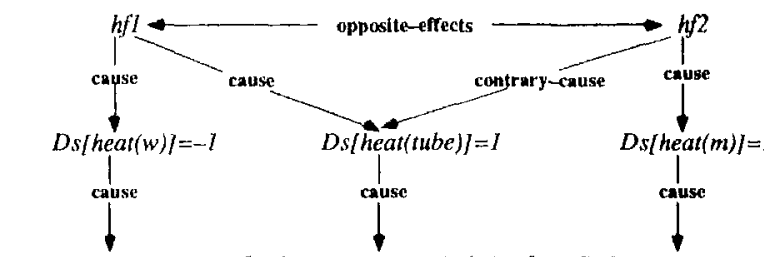

Ds/temperature $(w)]=-1$

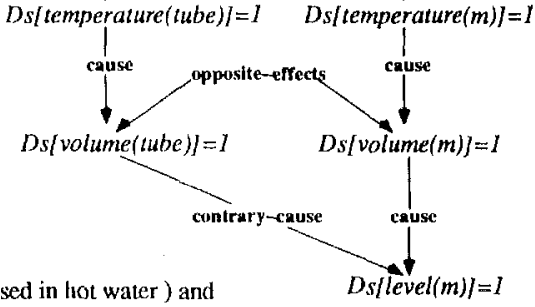

fig I : An example of physical system (a thermometer immersed in lot water) and its behavioural descriplion with an influence graph

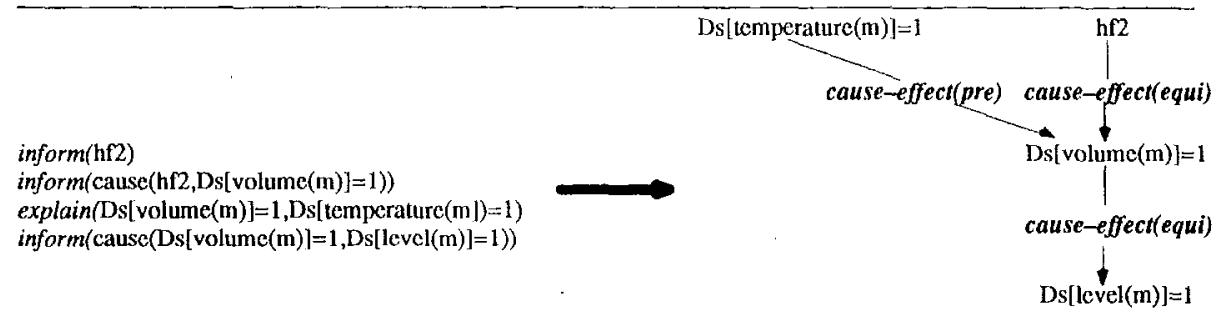

Il y a transfert de chaleur du tube vers le mercure. En conséquence, le mercure se dilate, car sa température augmente. Celte dilatation provoque la hausse du niveau de mercure.

(There is heat flow from the tube to the mercury. As a consequence, the mercury cxpands, for its temperature rises. This expansion causes the mercury level to rise.)

fig 2 : Application of the causal chain strategy

inform(cause(hf1,Ds[temperature $(w)]=-1)$ ) inform(cause(hf $1, \mathrm{Ds}[$ temperature(tube) $]=1)$ ) explain(Ds[temperature $(w)]=-1, \operatorname{Ds}[$ heat $(w)]=-1)$ explain(Ds[temperature (tubc)] $=1, \operatorname{Ds}[$ heat $($ tube $)]=1$ )

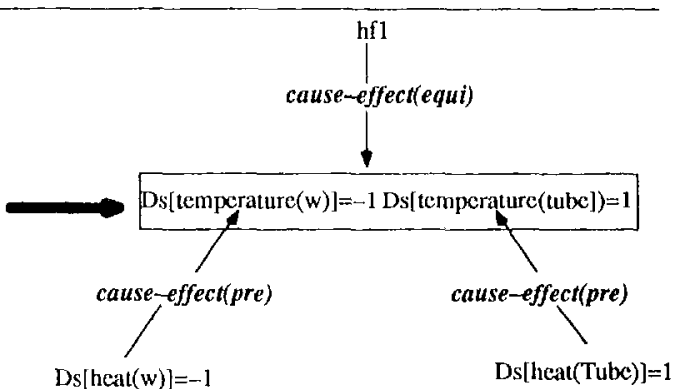

Il y a transfert de chaleur de l'eau vers le tube. En conséquence, la température de l'eau diminue. car sa quantité de chaleur diminue, et celle du tube augmente, car sa quantité de chaleur augmente.

(There is a heat flow from the water to the tube. As a consequence, the temperature of the water decreases, for its heat decreases and the temperature of the tube increases, as its heat increases.)

fig 3 : Application of the parallel strategy 
tion) and thus is governed by both general rhetorical principles and domain dependent considerations. It procecds as an incremental process and each step determines more precisely the relevant information. First the notion of themeobject, similar to global focus [Grosz 86] (and adapted to generation by Mc Kcown), is used to carry out a rough selection of relevant causal interactions: events which are not in (direct or indirect) causal interaction with any themeobject are ignored. The theme-object identification depends on the user interaction mode with the system. In the simplest cases, theme- objects can be identified with privileged properties of the physical system, such as outputs of an artifact. But in a dialogue system, an analysis of user queries is necessary.

Second, the selection is refined and communicative acts are selected according to user's heliefs and expectations cxpressed in his request. Especially, communicative acts on causal opposition relations are introduced in two kind of situation: (a) if a complete description of causal behaviour is required or (b) within a concessive strategy, which seems appropriate when the user focuses on an expected but yet unocccurred event. For example, in order to reply to the question "why doesn't the mercury level drop ?", causal opposition relations arc not ignored for it suggests that the user is interested with inhibited influences which might have caused a mercury drop. Similar considerations are expressed in Mc Coy's shemata for correcting misconceptions about object classifications [McCoy 89].

\section{IV - TEXT ORGANIZATION}

Text organization deals with multiple communicative acts simultaneously. This is one of the main features of our approach for it provides the ability to impose a global structure on the text. Global coherence cannot be ensured if communicative acts are considered independently of one another. Additionally, there is not a one to one correspondence between communicative acts and sentences of the text. A single utterance of ten realizes multiple communicative acts and inversely a single act may be spread on several utterances. For example, sentence (1) may also be uttered so as to achieve the three following acts: informing the hearer of mercury expansion and mercury level rise, and that these events are causally related. This conception in language generation has been initiated by Appelt in the KAMP system [Appelt 85]. He showed how multiple illocutionary acts can be achieved in a single utterance.

Communicative acts are not directly mapped to linguistic forms. The textual structure is introduced as an intermediary level of abstraction betwcen the specified content and the text. It is mainly composed of texthal units, inter-clausal relations, which indicate how these units should be related, and order prescriptions.

\section{IV.I - Inter-Clausal Relations}

Several text generation systems use inter-clausal relations which have been introduced in textual linguistics under various forms [Grimes 75], [Van Djik 81], [Halliday 76].
Rhetorical Structure Theory (RST) [Mann \&'Thompson 87] is a significant approach of this trend and it has been partially used in text planning systems [Hovy 88], [Moore \& Swartout 89]. In RST, the structure of a text is obtained by composition of rhetorical relations expressing various kinds of dependencies, sometimes semantic ("subject matter" relations in RST terminology), sometimes purely thetorical (presentational relation in RST). In contrast, in our approach the intended rhetorical effects are considered as communicative goals and are specified at the content specification level. As a consequence, inter-clausal relations involved in our system are exclusively semantic. Furthermore, they are characterized in a way similar to the paratactic/hypotactic distinction of rhetorical predicates [Grimes 75] so that they can take different communicative forms. Under the equiponderant form, the same interest is assigned to the arguments of the relation while under the preponderant form, one of them is presented as more central.

Arguments of relations are composed of textual units, which are semantic 1ranslations of conceptual entities. An argument may be simple (limited to a single textual unit) or complex (composed of several textual units).

At present our work on inter-clausal relations focuses on causal and adversative relations (i.c. on relations involved in the expression of our causal primitives).

\section{I.1.1 - Causal Inter-Clausal Relations}

In the cause-effect inter-clausal relation, events denoted in the first argument are presented as being the cause of events denoted in the second. According to the communicative form (equiponderant or preponderant) of the relation, different types of communicative acts may be achicved. The following elementary transitions (in a simplified form) both lcad to the introduction of a cause effect relation (in PROLOG notation, arguments in capitalized letters represent variables):

$$
\begin{aligned}
& \text { transition(inform of a causal link, } \\
& \text { inform(cause(C,E)), } \\
& \text { cause-effect(equi,C,E)). } \\
& \text { transition(explain with a causal link, } \\
& \text { (explain }(\vec{C}, \bar{E}) \text {, } \\
& \text { cause }\{C, E)\} \text {. } \\
& \text { cause-effect(pre, C, E))). }
\end{aligned}
$$

But there is not necessarily a direct correspondence between relations of conceptual level and relations of textual level, as in the above transitions. Hence, the following transition may hold in some context, even if no causal interaction exists between on the one hand $\mathrm{C} 1, \mathrm{E} 1$ and on the other hand $\mathrm{C} 2, \mathrm{E} 2$ :

$$
\begin{aligned}
& \text { transition(inform of muitiple_causal links, } \\
& \text { (inform(cause }(C 1, E \overline{1})) \\
& \text { inform(cause }(C 2, E 2))]_{,} \\
& \text {cause-effect(equi, }(C 1, C 2],[E 1, E 2])
\end{aligned}
$$




\section{IV.1.2 Adversative Inter-Clausal Relations}

As mentioned in section $I$, behavioural description of a physical system contains not only causal links between events but also causal opposition relations. These relations are often phrased in natural language with concessive connectives (but, though, however, ...) which, following Piaget [Piaget 78], express a discordance between cause and effect. The sentences (3) and (4) illustrate respectively expressions of contrary-cause and opposite-effects relations:

(3) Le tube se dilate mais le niveau de mercure monte.

(The tube expands but the mercury level rises.)

(4) Le tube se dilate mais le mercure se dilate aussi.

\section{(The tube expands but the mercury expands too.)}

The study of linguistic connectives within the linguistic Theory of Argumentation [Anscombre\& Ducrot 83], [Anscombre 84] and especially the French connective mais (but) has showed that two kinds of semantic opposition may be expressed by concessive connectives: direct opposition, where one of the two linked propositions is pres. ented as an argument for a conclusion opposed to the other proposition, and indirect opposition, where the two propositions are presented as arguments for opposite conclusions. (3) conveys a direct opposition since the conclusion (the mercury level drop) aimed by the first proposition is opposed to the second one (the mercury level risc) and (4) conveys an indirect opposition since the two propositions aim at opposite conclusions (mercury level drop and mercury level rise).

In order to represent these semantic oppositions, we have introduced the adversative inter-clausal relations directopposition and indirect-opposition. Acts of informing on causal opposition relations are then realized by means of these adversative relations:

$$
\begin{array}{r}
\text { transilion(inform_of_a_contrary_cause, } \\
\text { inform(contrary-cause }(C, E)), \\
\text { direct-opposition(pre, } C, E)) .
\end{array}
$$

transition(inform_of_opposite effects, inform(opposite-effects $(C 1, C 2))$, indirect-opposition (equi,Cl,C2)).

But also concessive acts, laking advantage of the concessive nature of adversative relations:

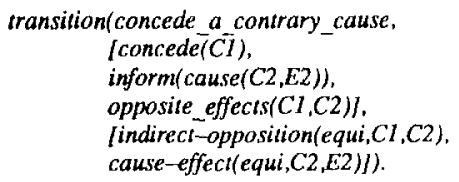

As we discussed earlier, communicative acts should not te examined independently of one another if one wants to produce well-structured texts. A local transition represents a possible treatment of a limited number of communicative acts at textual level. Hence, choice and application of local transitions are governed by discourse strategies. They determine transitions which may be applied after an analysis of the overall set of communicative acts. In particular, they exploit the underlying conceptual structure of communicative acts. For causal explanations, the mainly used strategies are the following:

- Causal Chain Strategy: If the underlying conceptual structure is a causal chain and communicative acts are of the inform or explain type then follow causal order and apply transitions inform_of a causal link and explain_with_a_causal_link (cffig 2).

- Parallel Strategy: It is a form of parallelism rhetorical "figure" which may be used when the underlying conceptual structure is composed of two causal chains with a common initial cause. This strategy also exploit the causal order and transitions inform_of common. cause_link and inform_of multiple_causal_links hold the highest priorities (cffig 3 ).

$$
\begin{aligned}
& \text { transition(inform_of_common cause_links, } \\
& \text { [inform(cause }(C, E)) \text { ), } \\
& \text { inform( cause(C,E2))], } \\
& \text { cause-effect(equi,C, }[E 1, E 2])
\end{aligned}
$$

- Concessive Strategy: This strategy deals with concessive acts which involve causal opposition rclations.

A preferential order is suggested: the conceded fact precedes the other units.

Priorities are assigned to the strategies so that confliets can be solved.

\section{IV.3 - Textual Structure Segmentation}

The purpose of the segmentation task is to delimit the content of the sentences which will constitute the final text. The determination of a sentential content involves several heuristics. Some of them aim at increasing the scope of the sentence while others aim at reducing it. One of the main heuristics deals with the communicative nature of interclausil relations: since preponderant relations often appear in subord inate forms at syntactic level, equiponderant relations are privileged. Hence, delimitation of sentential content starts with the choice of equiponderant relations. The content is then completed with preponderant relations. In addition, the number of textual units of a sentence is limited and introduced relations may be removed from the sentential content if it contains too many textual units. The segmentation is also constrained by the conceptual nature of information units. For instance, an initial cause may be realized in a single sentence. 


\section{V-SURFACE GENERATION}

The text planner is independent of the surface generation component. (except the stylistic adjustment task). Two different types of sentence generation approaches have been examined.

Our main approach aims at coupling the text planning system with a generic sentence generator [Fournicr 91] based on the Meaning-Text Theory [Mel'cuk 88]. In the Meaning-Text Model, the input representation is a semantic network enriched with several structures, mainly the bipartitions theme/rheme, asserted/presupposed and given/new. The construction of semantic networks is based on a conceptual-scmantic dictionary, which specifies correspondences between concepts and semantic definitions. The $g i$ ven/new structure has already been integrated in text generation systems (e.g. [Nirenburg 88]). It denotes specific linguistic constructions and will constrain choices during realization. For instance, a predicative structure will be rather realized as an assertion if it belongs to the new part or as a nominalization if it belongs to the given part. This characterization of semantic representations plays a crucial role from a textual perspective. The generation process must be sensitive to progression of new information through the text, even at the realization stage. In this spirit, distribution of semantic units among given and new parts is carried out with negard to conceptual units introduced in previous sentences. Predicative structures of the semantic network whose referents have been previously cvoked arc systematically marked as given and the others as new. Once the semantic representation is built (only the scmantic network and the giveninew structure have been considered), the realizer produces surface forms and paraphrases may be generated, essentially by lexical substitutions.

The second approach we are experimenting relieson equivalence classes between sentence structures, which may be compared to Discourse Grammars described in [Danlos 85]. An equivalence class represents potential realizations of a kind of semantic content. Our investigation is currently limited to classes related to causality. We have defined classes for expressing causal and opposition relations. This categorization of linguistic expressions provides a suitable basis for stylistic adjustment mechanisms. Syntactic and lexical constraints arising from stylistic considerations govern the choice of sentence structures within classes. In particular, we have implemented a "principle of stylistic variety" which prevents excessive use of similar syntactic constructions. This principle is particularly useful in causal explanation generation because concepts involved are of similar types. To illustrate this point, let us consider the text in figure 2. When generating the last sentence, stylistic adjustment recommends the realizer to avoid coordinative structures (with connectives such as thus, consequently, ...) since such a construction has been used in the previous sentence. A structure with a verb conveying causality (c.g. 10 cause, to result in, ...) is then selected.

\section{VI-RELATED WORKS}

Our system benefits from a number of earlier works on language generation, as those we have mentioned along the above sections. We will focus the comparison on two particular points. The first concerns the overall architecture and modularization in text generation systems; the second deals with text planning applied to causal explanation gencration. Text planning and surface generation have often been conceived as separated components. But separation of tasks is not always extended within text planning. For example, rhetorical schemata used in TEXT [McKeown 85] specify in the same structure not only information units which may be introduced but also their orders in the final text. Other systems as GOSSIP [Carcagno \& Iordanskaja 89 ] and PAULINE [Hovy 88b] have isolated content determination from text structuring. The approach described in this paper also proposes a clear separation of the content specification and the textual organization skills. The main motivation behind this division is that it is convenient to decide what to say (more preciscly, to set (almost) all communicative goals to achieve) before taking any organizational decisions. Hence, content specification proceeds regardless of structuring considerations. However, we think that a more cooperative interaction between these two major components is necessary to allow goal revisions at the text organization level.

Our system can also be compared to TAlLoR [Paris 87] which generates natural language descriptions of physical devices. A significant advantage of TAILOR is its ability to combine structural and causal description strategies. Never theless, causal interactions are restricted to simple causal links and there is no attempt to explicit the roles they can play in discourse.

\section{VII - FUTHER DEVELOPMENTS}

First a better conceptual characterization of physical systems would contribute to improve the quality of causal explanations. We need a more precise description of causal intcractions which allows, for instance, to discern enablement conditions from other causal links.

With regards to text planning, number of extensions are possible. We intend to define strategies for structural deseriptions and also enhance the control mechanisms of discourse strategies. Furthermore, practical validation of the overall approach requires a larger coverage of communicative acts. Another interesting extension would consist in relating stylistic adjustment mechanisms to pragmatic features [Hovy 89] in order to strengthen context sensitivity.

\section{Acknowledgments}

I would like to thank Corinne Fournier and Marie-Christine Escalier for their comments on earlier versions of this paper. 
- Anscombre, J.C. "Grammaire traditionnelle et grammaire argumentative de la concession", Revue internationale de philosophie, 155, 1984.

- Anscombre, J.C. \& Ducrot, O. L'Argumentation dans la langue, Bruxelles, Mardaga, 1983.

- Appelt, D.E. "Planning Natural Language Referring Expressions", Artificial intelligence, 25, 1985.

- Carcagno, D. \& Iordanskaja, L. "Content Determination and Text Structuring in GOSSIP", 2 nd European Workshop on Natural Language Generation, 1989.

- Cerbah, F. \& Fournier, C. \& Raccah, P.Y. "Qualitative reasoning and Argumentation: $A$ study of some affinities when Generating Causal Explanations", 1st Workshop on Qualitative Reasoning and Decision Support Systems, 1991.

- Cohen, P. \& Perrault, C.R. "Elements of a Plan Based Theory of Speech Acts", Readings In Natural Language Processing, Morgan Kaufman Publishers, 1986.

- Danlos, L. Génération automatique de textes en langues naturelles, Masson, 1985.

- De Kleer, J. \& Brown, J.S. "A Qualitative Physics Based on Confluences", Artificial Intelligence, 24, 1984.

-Elhadad, M. \& Mc Keown, K. "Generating Connectives", COLING 90.

- Forbus, K.D. and Gentner, D. "Causal Reasoning about Quantities", Proceedings of the Fifth Annwal Conference of the Cognitive Science Society, Lawrence Erlbaum Associates, Inc, 1983.

-Forbus, K.D. "Qualitative Process Theory", Artificial Intelligence, 24, 1984.

-Forbus, K.D. "Qualitative Physics: Past, Present, and Future", Exploring Artificial intelligence, Howard Shrobe (eds). Morgan Kaufmann Publishers, Inc, 1988.

- Foumier, C. "Un générateur de tex tes fondé sur le modèle Sens-Texte", Technical Report Dassault Aviation, 1991. - Grimes, J.E. The Thread of Discourse, Mouton, 1975.
- Grosz, B.J. \& Sidner, C. "Attention, Intention and the Structure of Discourse", Computational Linguistics, 1986. - Halliday, M.A.K. \& Hasan, R. Cohesion in English, London, Longman, 1976.

- Hovy, E.H. "Planning Coherent Multisentential Text", 26th ACL, 1988 .

- Hovy, E.H. "Pragmatics and Natural Language Generation", Arlificial incelligence, 43, 1989.

- Jézéquel, P. \& Zimmer, L. "SQUALE : manuel d'utilisation", Technical Report Dassault Aviation, 1992.

- Mann, W.C. \& Thompson, S.A. "Rhetorical Structure Theory: A Theory of Text Organization", ISI/RS-87-190, 1987.

- McCoy, K.F. "Generating Context-Sensitive Responses to Object-Related Misconceptions", Artificial Intelligence, 41, 1989.

- Mckeown, K. Text Generation, New York, Cambridge U. Press, 1985

- McKcown, K. \& Swartout, W. "Language generation and explanation", Advances in Natural Language Generation, Zock and Sabah (cds), London, Pinter, 1988.

- Mel'cuk, I. Dependency Syntax: Theory and Practice, SUNY, 1988.

- Moore, J. \& Swartout, W. "A Reactive Approach to Explanation", IJCA/ 89.

- Nirenburg, S. \& Nirenburg, I. "A framework for lexical sclection in natural language generation", COLING 88 .

- Paris, C.L. "Combining Discourse Strategies to Generate Descriptions to Users Along a Naive/Expert Spectrum", $I J$ CAI 87

- Piaget, J. Le Jugement et le raisonnement chez l'enfant, Delachaux et Niestle, 1978.

- Suthers, D.D. "Perspectives in Explanation", COINS Technical Report 89-24, 1989.

- van Dijk, T.A. Studies in the Pragmalics of Discourse, Mouton, 1981. 